\title{
El papel de las mujeres sobrevivientes en la construcción de paz en Colombia
}

\author{
The role of surviving women \\ in the construction of peace in Colombia
}

Wilman Preciado Valencia* Heidi Smith Pulido Varon**

\begin{abstract}
Resumen
El presente artículo reflexiona respecto a las contribuciones que hacen las mujeres sobrevivientes del conflicto armado a la paz en Colombia, a partir de dos categorías: la primera, explora la experiencia de las mujeres desde la perspectiva de la violencia patriarcal; la segunda, recoge los aportes que han hecho ellas a la construcción de paz. Entre otras conclusiones importantes se reconoce: cómo la violencia patriarcal contra las mujeres laceró su dignidad y pretendió minar su liderazgo político y social; cómo la acogida entre mujeres, no necesariamente vinculadas a estrategias institucionales de orden psicosocial, fortalecen su recuperación; y cómo las estrategias noviolentas pretenden movilizar a la sociedad colombiana a la solidaridad, y al reconocimiento de la otredad que representa la fragilidad de quienes han sufrido la violencia y demandan la consolidación de la paz.
\end{abstract}

Palabras clave: construcción de paz, estrategias noviolentas, experiencias de acogida, violencia patriarcal.

\begin{abstract}
This article reflects on the contributions that women survivors of the armed conflict make to peace in Colombia, from two categories, the first denominated women and patriarchal violence and the second, contributions of women to peacebuilding. The most representative conclusions are three: 1) it is recognized that patriarchal violence against women undermines dignity and sought to undermine their political and social leadership, 2) reception among women, not necessarily linked to institutional strategies of a psychosocial nature, strengthen the recovery of women, 3) non-violent strategies seek to mobilize society to solidarity, to the recognition of the otherness that represents the fragility of those who suffered violence, demand the consolidation of peace.
\end{abstract}

Keywords: peace building, patriarchal violence, reception experiences, non-violent strategies.

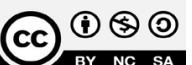

ReCibido: I5 DE JULIO DE 20I9 | APROBADO: I6 DE OCTUBRE DE 2019

Cómo CITAR ESTE ARTICULO

Preciado Valencia, W. y Pulido Varon, H. (2020). El papel de las mujeres sobrevivientes en la construcción de paz en Colombia. Collectivus, Revista de Ciencias Sociales, 7(1), 109-120. DOI: https://doi.org/10.15648/Collectivus.vol7num1.2020.2555

*Magister en Intervenciones Psicosociales. Facultad de Ciencias Sociales y Religiosas, Fundación Universitaria Claretiana, jefatura del Departamento de Humanidades, sede principal, Quibdó, Chocó. departamentohumanidades@uniclaretiana.edu.co

**Magister en Desarrollo. Facultad de Ciencias Sociales y Psicología, Universidad Católica Luis Amigó. Bloque 1-sala 41. heidi.pulidova@amigo.edu.co 


\section{Introducción}

El 24 de noviembre de 2016 se firmó el Acuerdo final para la terminación del conflicto armado en Colombia y para la construcción de una paz estable y duradera entre el Estado colombiano y la guerrilla de FARC-EP. En términos formales se dio por finalizada una confrontación bélica que supera los 50 años (cfr. Comisión Histórica del Conflicto y sus Víctimas, 2015, pp.13-14); para la sociedad colombiana esto significa el inició un nuevo desafío por alcanzar la paz, incorporando en lo cotidiano disposiciones para el perdón y la reconciliación, no solo hacia quienes han participado directamente del conflicto, sino también en todos los niveles sociales en los que han calado las lógicas de la violencia.

En efecto, es imprescindible entender que la consolidación de la paz en Colombia no se sujeta exclusivamente a la formalidad del Acuerdo o visiones que buscan el silencio de los fusiles, la búsqueda de la justicia o el fin de la violencia estructural sino que demanda una sociedad reflexiva y en capacidad de valorar la importancia del cuidado del otro, la no agresión, la vida misma, posiciones que se condensan en lo que algunos teóricos identifican como Paz Negativa y Paz Positiva (cfr. Muñoz, 2014, pp. 6-7; cfr. Ramos, 2016, p.514), desde las cuales se demarca la importancia de atender los problemas que se presentan en la vida cotidiana y la participación de los actores sociales en su resolución (cfr. Castaño, 2013, p.15; Bernardelli, 2014, p.6; Turriago, 2016, p.177).

Abrir la comprensión respecto a la construcción de paz y asumir el rol que juega la sociedad civil, (cfr. Rettberg, 2013, p.6) representa una apuesta hacia lo que Muñoz (2014) denomina Paz Imperfecta, puesto que obliga a visibilizar las lógicas de poder que sostienen la violencia y el reconocimiento de los procesos que de manera anónima se han desarrollado en los territorios locales buscando un mínimo de condiciones para la sobrevivencia, la dignidad humana y la resistencia a la violencia en medio del conflicto (cfr. Muñoz, 2004, pp.31-32).

Las mujeres, principalmente aquellas que sobrevivieron al conflicto en condiciones complejas para la recuperación de su proyecto de vida, constituyen un foco importante de atención, pues sus iniciativas representan procesos de resistencia noviolentos frente a las dinámicas y actores del conflicto, así como alternativas de acción política que de forma directa o indirecta contribuyen a la construcción de paz en el país (cfr. Rettberg y Quishpe, 2017, p.14).

Si bien es cierto, la violencia de corte patriarcal impuesta sobre las mujeres durante el conflicto dejó problemas de orden psicosocial que operan como limitantes del rol femenino en la construcción de paz, se presenta como significativo el quiebre de las lógicas del dominio patriarcal generador de violencia; primero, porque las mujeres no salieron de la violencia con sentimientos de revancha o venganza, sino con pretensiones de reconciliar al país (Villa, Avendaño, y García, 2017; Binazzi, 2019 ), y segundo, porque las mujeres resignifican el lugar de la víctima que perpetua estigmas y relega a lo pasivo, invisibilizando su lugar como constructoras de nuevas realidades sociales y políticas (ob. Cit, p.41).

Esa posición de las mujeres es política en la medida que sugiere apertura, ejercicio de libertades, transformación y reivindicación en lo cotidiano, entendiendo que la política es parte constitutiva del quehacer humano y busca la libertad humana. De acuerdo a Tatián (2006):

Es la posibilidad de no ser esclavos (del amo, del patrón, del capitalista, del militar, del burócrata, del mercado [...]). O también -positivamente dicho-, política es la construcción colectiva de la libertad, es la institución de la libertad pública [...] La política es lo que tiene que ver con la libertad y la dominación -y por añadidura con el poder. (p.105) 
Esa construcción colectiva de libertad está impregnada en los actos de las mujeres, que muestran un compromiso ciudadano por transformar las estructuras de dominación y posibilitar relaciones marcadas por la libertad de las personas.

La construcción de paz pasa por visibilizar y potenciar los actos políticos de las mujeres que sufrieron las vehemencias del conflicto armado y esto se logra, cuando el Estado asume su responsabilidad en las leyes y las políticas públicas (cfr. Aguilar, 2015, p.24) que permitan a ellas sanar las heridas dejadas en el conflicto a la par que posibilita condiciones dignas para que reconstruyan su vida y tengan garantías para la realización de propuestas que coadyuven a la consolidación de la paz. Asimismo, según el Centro Nacional de Memoria Histórica, incluye:

Un trato equivalente en cuanto a dignidad humana; respeto a las diferencias; acceso a la justicia en condiciones de paridad; igualdad de oportunidades para desenvolverse y ser escuchadas e incluidas en los procesos de reconstrucción de la memoria histórica, la justicia y la reparación integral; garantías a sus derechos patrimoniales; y garantías de respeto a sus derechos sexuales y reproductivos. (Centro Nacional de Memoria Histórica, 2011, citado por Cadavid, 2014, p.315)

Si bien, el Estado ha abierto posibilidades para que las mujeres puedan sanar, encontrar nuevos sentidos a la vida y potenciar sus capacidades para construir un mejor país, el interés de este escrito es mostrar como por fuera de la institucionalidad, las mujeres trabajan en favor de una paz estable y duradera, desde un encuentro con la otra donde se da acogida, compañerismo, confianza, festividad, tradición, alegría y complicidad; elementos significativos para sus procesos de reparación, perdón y reconciliación; todos ellos decisivos en un proceso de construcción de paz.

En consecuencia, el presente texto se estructura bajo tres apartados: el primero titulado violencia de corte patriarcal durante el conflicto, el segundo, experiencias de acogida que sanan y restablecen la dignidad femenina, y el tercero, estrategias noviolentas para la construcción de paz en Colombia.

\section{Violencia de corte patriarcal durante el conflicto}

En el conflicto armado colombiano, el patriarcado se impuso con acciones violentas contra las mujeres dejando impactos que contribuyeron a profundizar las brechas entre hombres y mujeres, sometiéndolas en contra de su voluntad a toda clase de violencias. En efecto, "las mujeres han sufrido diferentes vulneraciones de Derechos Humanos, como violencia sexual, desplazamientos forzados, asesinatos, amenazas, desapariciones forzadas y otros atentados contra la integridad física" (Garrido y Vidal, 2018, p.126).

Esta visión anquilosada del poder generó terror y toda clase de sometimiento de las mujeres hasta establecer un orden patriarcal y despótico en los territorios que lograron controlar (cfr. Centro Nacional de Memoria Histórica, 2011, p.18).

La misma fuente reconoce, que no solo las mujeres sufrieron la violencia de género, pero fueron las víctimas principales durante el conflicto armado, dado que esta tiene una múltiple representación, por un lado, humillación para la víctima, reforzamiento del poder en el victimario y zozobra para al resto de la población:

En el interminable listado de vejaciones, humillaciones y torturas que las mujeres padecieron, la violencia sexual ocupa un lugar destacado, por ser uno de los hechos crueles más frecuentes, y que reúne unos significados más complejos en cuanto a lo que representa de demostración de poder para el victimario y de abuso y humillación para quien la sufre (Informe Proyecto Interdiocesano de Recuperación de la Memoria Histórica, Guatemala: Nunca Más, 1998, citado por el Centro Nacional de Memoria Histórica, 2011, p.19). 
Ahora bien, la violencia contra la mujer se ejerció no solo desde el acceso carnal violento, sino que se extendió hacia dimensiones simbólicas, relacionales y afectivas que derivaron en prácticas cotidianas ligadas a la dominación patriarcal (cfr. Andrade et al., 2017, p.296). Así, la prostitución forzada, el reclutamiento, la violencia intrafamiliar, las violencias sexuales, entre otras, sirvieron para humillar al adversario, intimidar a la población y coaccionar a las mujeres en su rol social, político y comunitario.

Los patrones de violencia contra las mujeres ejercidas durante el conflicto armado han estado presentes en la sociedad, al mismo tiempo que se ha desplegado la violencia política (cfr. Castrellón y Romero, 2016, p.77). El impulso a la dominación, el control, los sesgos de género entre otros elementos, permean la subjetividad de las comunidades y mantienen lógicas patriarcales que operan desfavorablemente para una paz estable y duradera.

Así las cosas, no es gratuito que el Acuerdo de paz esté permeado por una perspectiva de género, la cual reconoce problemas derivados de la desigualdad de derechos entre hombres y mujeres que resultan importantes atender (cfr. Acuerdo final para la terminación del conflicto y la construcción de una paz estable y duradera, 2016, p.231). Efectivamente, la consecución de una paz estable y duradera, además de requerir el compromiso formal entre el Estado y las FARC-EP, demanda atender las relaciones asimétricas de la sociedad colombiana donde confluyen injusticias que históricamente han sido caldo de cultivo para las diversas violencias contra las mujeres.

En el Acuerdo de la Habana, la paz es considerada un derecho superior, garantista de los demás derechos y deberes de los colombianos que requiere ahondar en asuntos como la otredad y las asimetrías de género, que adquieren características particulares en el rostro femenino. Ejecutar los componentes del Acuerdo en los territorios y otorgar a las mujeres un lugar privilegiado, tanto en su implementación, como en el soporte a la construcción de paz es un paso orientado a ello. Por lo demás, el Acuerdo reconoce a la mujer como sujeto de derecho y prioriza la protección de su integridad con el fin de evitar la repetición de la violencia (ob. Cit, p.12).

Al considerar a las mujeres sujetos de derecho y al establecer las condiciones para un liderazgo enfocado a la construcción de paz, se da un paso hacia el respeto y valoración del otro, denotando un Estado y una sociedad abierta a la paz. Al fin y al cabo, el respeto a la integridad de la mujer y la garantía de sus derechos encaminan al país por una cultura de paz que implica reconocer, reparar y desterrar los abusos permitidos en el conflicto y perpetuados en distintos escenarios donde se entretejen las relaciones humanas, porque no habrá paz bajo el dominio y la violencia ejercidos contra las mujeres en ámbitos públicos y privados (cfr. Céspedes-Báez, 2018, p.95).

Por eso, Villa, Avendaño y García (2017) sostienen que, la eliminación del patriarcado de la sociedad debe transformar el rol ocupado por la mujer, tradicionalmente relegado al ámbito familiar, a lo privado y lo doméstico, en los cuales es cosificada y devaluada. De acuerdo con los autores mencionados:

Implica además el cambio en roles tradicionales que ocupaba la mujer en la sociedad patriarcal y machista de la que hace parte, para empezar a construir escenarios que llevan de lo privado a lo público, con lo cual se movilizan también las formas de relación en la familia y la comunidad, especialmente con los hombres. (ob. Cit, p.6)

Por consiguiente, la priorización del desmonte del patriarcado de la sociedad es uno de los focos en el cual las mujeres vienen trabajando. Esto equivale a procesos de resistencia a la violencia, para fortalecer en la cotidianidad el respeto y la autonomía, asuntos necesarios para esta nueva etapa del país donde se asume la paz desde el respeto por el otro, por las mujeres (cfr. Silva, 2017, p.67). 
En cualquier caso, el desmonte del patriarcado como condición de la construcción de paz, no sólo debe ser un trabajo impulsado por las mujeres, sino que toda la sociedad, el Estado y sus instituciones tienen la responsabilidad de promoverlo, asunto que se complejiza, pues el patriarcado es un eje fundamental en el sistema hegemónico de dominación y se anuda a dimensiones económicas y de poder, donde el cuerpo y la dimensión femenina, se anula, se utiliza y degrada.

Dado lo anterior, es comprensible que los avances del enfoque de género aun tengan una introducción mínima en diferentes áreas. En el caso particular de la jurisprudencia, el enfoque de género apunta a garantizar el equilibrio de los derechos para toda la población y en particular, para que las mujeres afectadas por el conflicto armado gocen de los derechos contemplados garantizados en el Acuerdo de paz (cfr. Bustamante, 2015, p.21). En tal sentido, Bustamante (2015) plantea la reformulación del discurso patriarcal en la jurisprudencia colombiana para establecer un orden justo y garantizar los derechos de las mujeres:

Colombia padece el conflicto armado hace más de seis décadas; de hecho, durante mucho tiempo la violencia sobre el cuerpo de las mujeres no fue nombrada puesto que la jerarquización, producto de la sociedad patriarcal, solo veía como víctimas a los hombres; de hecho, la inclusión de las mujeres como víctimas de violencia sexual ha sido difundida principalmente por la jurisprudencia de los tribunales penales internacionales en la década del noventa. (p.31)

Por tanto, en la medida que se desmonte del derecho el sistema patriarcal, se avanzará en "la reparación de las mujeres víctimas desde una perspectiva que incluya su situación social, económica y política, para acercarnos a una verdadera transformación de las estructuras sociales patriarcales" (Castrellón Pérez \& Romero Cristancho, 2016). Reparación que posibilitaría la sanación de las heridas y secuelas que dejó la violencia armada, para que las mujeres sigan fortaleciendo sus capacidades y liderazgos a favor de la implementación de los Acuerdos de paz en sus territorios.

\section{Experiencias de acogida que sanan y restablecen la dignidad femenina}

Este apartado presenta cuatro estrategias psicosociales enfocadas a la sanación de mujeres que sufrieron la violencia durante el conflicto armado; las estrategias son diversas y realizadas por organizaciones de mujeres y la Unidad de Víctimas. Ni estas organizaciones, ni la entidad gubernamental, ni muchos menos sus estrategias agotan las iniciativas que se realizan en beneficio de ellas y de la paz en Colombia. En Colombia como lo registra Villa (2016) hay diversas iniciativas y organizaciones donde las mujeres son protagonistas de su propia recuperación y de los aportes que hacen para acabar el conflicto armado (cfr. Villa, 2016, p.7).

Así pues, las estrategias que se expondrán por su diversidad de enfoque y abordaje concentran parte de las formas como se viene asumiendo el tema de atención a las mujeres y su posterior participación en la resolución de los problemas personales y comunitarios provenientes del conflicto armado (Magallón, 2004).

La Unidad de Víctimas (2018), por ejemplo, creó la Estrategia de Reparación a Mujeres Víctimas de Violencia Sexual que pretende que las mujeres avancen hacia el reconocimiento y protección de derechos personales y comunitarios, fortalezcan espacios para el diálogo y el fortalecimiento de proyectos de vida, contribuyan a la reparación y construcción de paz. Al mismo tiempo, la estrategia es un vehículo para que las mujeres se escuchen y creen redes de solidaridad.

Igualmente, (Heridas \& Mujeres, 2016) implementa la estrategia La Narrada, bajo la cual, las mujeres logran develar las atrocidades de la guerra, se animan a denunciar y a abogan por la justicia. 
Dicha estrategia destaca:

En el encuentro con otras, la escucha conmovida, la palabra acogedora, el abrazo solidario, el alimento compartido, el conocimiento ancestral, la música y el tejido, acompasaron relatos de los dolores y horrores que dejó la violencia. Sólo entre mujeres era posible nombrar lo innombrable, aquello que todas, a su manera, habían vivido. (p.6)

Para esta corporación las mujeres en la espontaneidad de los relatos cuentan la violencia y al contar se produce en ellas una especie de catarsis que no se queda en el pasado doloroso, sino que cobra una dimensión trascendental; contar, narrar, conversar, eleva el espíritu hacia fuentes renovables, para:

Recuperar la simple conversación que nace en la intimidad de las cocinas, en el frescor de los patios, alrededor de un café. La espontánea y vital conversación entre mujeres, que adquiere una dimensión trascendental si se trata de contar experiencias de violencia. (ob. Cit, p.8)

Así, la narración es conexión espiritual y da a quien cuenta la historia, un sentido transcendente de la vida, puesto que, se llega a comprender que no todo se ha acabado. De hecho, el narrar conecta lo más profundo y rompe lo efímero, es lo que (Heridas \& Mujeres, 2016) nombra recuperación transformadora, ya que devuelve la alegría, la confianza y la esperanza (cfr. Ob. Cit, p.17)

Sin embargo, la corporación enfatiza que la conexión no es efímera, sino vinculada a la realidad y a los hechos políticos, en el sentido que ejercen actos que producen un "deseo de libertad (o, de manera reversible, reacción contra toda forma de esclavitud); interés e intervención en lo público, orientados a transformar estados de situación independientemente de todo beneficio privado" (Tatián, 2006, p.106). Entonces son actos en función de poner en paz al agresor y al agredido, y al mismo sistema donde se sostiene el conflicto.

En esa misma línea, ONU Mujer (2016) crea una experiencia de sanación basada en las representaciones simbólicas del cuerpo, por medio de la cual las mujeres logran representar y liberarse de la violencia. A la par, se comprenden, restablecen la confianza, se solidarizan entre ellas y mueven a la sociedad hacia la empatía con quienes fueron afectados por la violencia del conflicto.

En esta estrategia psicosocial de sanación simbólica el cuerpo desempeña un rol importante que transmite paz, ya que el símbolo "en lo que tiene de externo, revela una realidad interna; en lo que tiene de corporal, una realidad espiritual; en lo que tiene de visible, lo invisible" (Lurker, 1992, citado por Solares, 2008, p.43). El cuerpo en escena remite a experiencias que no son vánales, y a veces no perceptibles a primera vista, pero que están ahí, envían mensajes.

Para ONU Mujer (2016) en el performance, el cuerpo de las mujeres devela e invita al público a romper el silencio, a compartir lo sucedido, la causa, hacerse solidario (ob. Cit, p.97). Bajo esta perspectiva, puede comprenderse el cuerpo símbolo propiciador de reconciliación pues en sus actos, muestra la causa propia para comprometer al otro. El acto, como lo expresa Butler (2016), deja de ser solitario para hacerse solidario (Cfr. Butler, 2016, p.306), de modo que el mismo cuerpo que ha sido agredido y sometido, logra denunciar la violencia e invita a recuperar la dignidad y la búsqueda de justicia que se necesita para que el otro sea reconocido, no vencido ni eliminado.

Por otra parte, el símbolo, que se representa con el performance del cuerpo -como lo expresa (ONU Mujer, 2018)- "tiene un componente de liberación, de elaboración, desprendimiento, como si las mujeres estuvieran por fin sacando de su cuerpo, de su intimidad, el poder del agresor que allí había quedado anclado" (p.26). Así pues, no solo se liberan del pasado violento, sino que el cuerpo en performance, "expresa un deseo de renacer, de transición del dolor y la guerra hacia la alegría y la paz" (ob. Cit, p.26). De ahí, puede decirse que las mujeres vuelven a la vida empoderadas de su propia historia. 
Así mismo, se fortalece valores, derechos y expresiones artísticas: se recupera la confianza, la cercanía, la ternura, la solidaridad, la expresividad, el arte, la memoria, la justicia, la política, el derecho a disentir, a exigir y a proponer, a proponer acciones en favor de la construcción de paz, o como lo recoge el (CNMH, 2017) por medio del siguiente relato: "sanar significa para nosotras poder sentirnos bien con nosotras mismas y con quienes nos rodean, poder expresarnos, abandonar el resentimiento, recuperar la autoestima, la confianza en nosotras mismas y el respeto por los demás" (p.268).

La cuarta estrategia psicosocial de sanación, el perdón y la reconciliación Villa (2016) la asume de las experiencias de mujeres de los departamentos de Córdoba, Bolívar y Antioquia, de éste concretamente, de la Corporación AMOR, para esta entidad al sanar por medio del perdón se corta la cadena de violencia que se reproduce constantemente, de ahí que los protagonistas de la violencia hombres y mujeres:

En diversas regiones del país, todos en una lógica noviolenta, y la mayoría de ellos con propuestas de perdón y reconciliación, son una prueba de los procesos y dinámicas que desarrolla la gente para construir paz desde abajo, en contraposición a los discursos que legitiman la guerra, generando escenarios para la reconstrucción del tejido social. (p. 43)

Villa (2016) expone la desarrollada por La asociación de mujeres del Oriente Antioqueño (AMOR), “cuyas bases sociales son sus organizaciones y redes en los 23 municipios del Oriente y lideresas destacadas del nivel municipal y regional, sensibles y comprometidas con los asuntos de las mujeres y la equidad de género" (Mujeres, 2013). Para esta asociación que se dedica al cuidado de la vida, la reconciliación y participación política, el perdón se ofrece libremente, sin cohesión, ni mucho menos como afrenta a la dignidad de las mujeres (ob. Cit, p.7). Éstas, que, "experimentaron la ruptura del tejido social, la zozobra, el miedo, el dolor, la rabia, la ira, el resentimiento y el odio por las pérdidas y rupturas, por las humillaciones padecidas" (ob. Cit, p.43). Buscan con sus acciones hacer las paces por medio del perdón ofrecido libremente.

De acuerdo con el autor citado, la violencia padecida por las mujeres, sus familias y en las mismas comunidades las movilizó a frenar la violencia con estrategias que llevaran a la reconciliación en la región. Este proceso, según Villa (2016) propició “el reclamo de una negociación política que condujera al final de la guerra, exigir el respeto a la vida y la generación de espacios locales de reencuentro y reconstrucción de las relaciones cotidianas en las mismas comunidades" (p.8).

Así pues, con el apoyo de las ONG avanzaron en un proceso de sanación que condujera a la búsqueda de la paz sin agresión, donde las mismas mujeres que habían sido agredidas se convirtieran en promotoras psicosociales en función de orientar a otros y otras hacia la recuperación, como así lo recoge Villa (2016) al narrar las formas como se dio el proceso:

\footnotetext{
En este proceso se formaron como promotoras psicosociales para el acompañamiento a los afectados por el conflicto armado y se estableció una metodología de grupos de apoyo mutuo, que contaban con la presencia de 15 personas, en promedio, durante mínimo un año de proceso. Se generó, entonces, la posibilidad del encuentro entre personas de diferentes edades y género en las diversas localidades, que pudieron mirarse al rostro, compartir las experiencias de dolor, rabia, miedo, deseos de venganza, en una lógica que iba más allá de la victimización, la patologización de sus reacciones emocionales o sus comportamientos defensivos, y de los tipos de violencia sufrida según los actores perpetradores. (p.8)
}

Ahora bien, como resultado de este proceso villa (2016) rescata, a) la recuperación emocional que concientizó a las mujeres y rompió la lógica de la victimización para integrarse con vitalidad a la comunidad con alternativas en pro de la reconciliación. b) Dichas alternativas basadas en el restablecimiento de "lo humano que fue roto en la gente, en las personas, en las comunidades" (p.19). C) Por el cual "se recuperaron confianzas, se volvieron a tejer solidaridades y se generó un escenario micropolítico de reconciliación social entre personas de la comunidad, más allá de las dinámicas macropolíticas de la guerra y la paz" (p.9). 
De todo este proceso, Villa (2016) concluye que "emerge el perdón como un nuevo dispositivo socioemocional que tiene una dimensión personal, social, política, espiritual y cultural, que tiene el poder noviolento de generar transformaciones profundas en la lógica de los conflictos atravesados por violencia" (p.10). Lógica que, atravesada por el perdón y la reconciliación, permite el surgimiento de lo emergente, esto es, el "compromiso para reconstruir tejidos de solidaridad y apoyo, marcados por el interés por el otro, la preocupación por lo comunitario y el restablecimiento de relaciones donde la vida recupera su sentido" (p.10).

Ahora bien, la Estrategia de Reparación a Mujeres Víctimas de Violencia Sexual, realizada por la Unidad de Víctimas se lleva a cabo en las regiones donde hay mujeres que fueron afectadas por el conflicto. La misma, está estructurada en tres momentos, cada uno incluye formación en torno a la reparación y se realizan estrategias psicosociales de sanación: el momento uno, identificado como acercamiento y orientación, busca restablecer la confianza entre las mujeres y el Estado; el momento dos, se conoce como perspectiva de derechos de las mujeres, aquí conversan previo a las iniciativas psicosociales de sanación y reflexionan sobre los derechos sexuales y reproductivos; el momento tres, implica un intercambio de saberes y actos simbólicos, cuyo conjunto sirve para apoyar la recuperación (Víctimas, 2018; Fouskas, Gikopoulou, Ioannidi, y Koulierakis, 2019). Algunas consecuencias positivas que salen de los encuentros la Unidad de Víctimas los presenta como, a) la creación de lazos entre las mujeres participantes, a medida que avanza el proceso se crean y se fortalecen lazos entre las mujeres y surge el compañerismo. b) La creación de espacios para hablar libremente, donde se dan procesos de descarga emocional. c) El empoderamiento de las mujeres a través de la formación en derechos, el cual también les ayuda para la reconstrucción de su proyecto de vida. (ob. Cit, p.26)

\section{Estrategias no violentas para la construcción de paz en Colombia}

Las estrategias colectivas de resistencias que promueven las mujeres pretenden movilizar a la sociedad colombiana hacia la solidaridad con quienes fueron violentados y con las comunidades que históricamente han sufrido la violencia (Meza, 2015, p.16), pues la construcción de un país en paz pasa por el papel activo que la sociedad civil pueda asumir y la solidaridad con quienes han sufrido durante el conflicto.

De acuerdo con Villa (2016) las acciones movilizadoras noviolentas en las que participan las mujeres sobrevivientes del conflicto armado colombiano están en función de conseguir, a) reconocimiento social en los contextos locales, b) reclamo de una negociación política para acabar la guerra, c) respeto a la vida, d) creación de espacios para el reencuentro y reconstrucción de relaciones con la comunidad, e) exigencia de derechos y desavenencia con la injusticia y explotación humana (p.8).

También, Ibarra (2015) citado por Sánchez y Rodríguez (2015) se refiere a las acciones movilizadoras noviolentas, de las mujeres que "pretenden lograr reconocimiento, desarrollo y protección de intereses y necesidades individuales o colectivas que ellos consideran que expresan el bienestar colectivo, la igualdad, la libertad, la justicia, la emancipación, o, en general, la dignidad humana" (p.158). Estas acciones noviolentas se realizan, como lo expresan los autores citados (2015) con estrategias "generalmente no convencionales - huelgas, manifestaciones, acciones no violentas o eventualmente violentas, entre otras. -, con un alto nivel de integración simbólica y unas nutridas formas de organización variable" (p.158). De hecho, el nivel de integración simbólica de las acciones noviolentas, al decir de Villa et al (2017) exaltan "las luchas de las mujeres para la construcción de paz y sus resistencias a la propagación de la violencia y la guerra en diferentes lugares del mundo, especialmente en el contexto del conflicto armado colombiano" (p.3). 
Asimismo, esas luchas reflejan la evolución que ha tenido el rol de la mujer de pasar de lo privado a lo público y en lo público a jugar un papel político en la sociedad y en particular en sus comunidades violentadas durante el conflicto armado. Al respecto, Villa et al (2017) afirman, la mujer "pasa de ser un bien privado, generador de vida y, susceptible de daño y control, para apropiarse de un rol político, desde el cual, desarrollan estrategias de cambio y trasformaciones sociales distintas a la eliminación sistemática del enemigo" (p.4). De hecho, al pasar de lo privado al campo de lo público aportan a la pacificación de la sociedad (cfr. Urrutia, 2017, p.3). Con estos aportes, se oponen al belicismo por parte del Estado, median en las confrontaciones bélicas, trabajan en favor de la verdad, la justicia y la reparación, hacen cabildeo ante las instituciones políticas, participación en espacios y movimientos políticos y se solidarizan entre ellas (cfr. Sánchez y Rodríguez, 2015, p.170).

Por supuesto, las estrategias noviolentas sobrepasan los altos niveles donde se toman decisiones a favor de la resolución de conflictos (cfr. Mesa, 2018, p.210), influyen en la vida cotidiana donde irrumpe la violencia, y sobre todo, en este nivel, las mujeres que sufrieron la violencia, por un lado, buscan concientizar a la población a favor de la paz, y por otro, plantean una idea de paz abierta a la participación de la población civil (cfr. Urrutia, 2017, p.3). Así pues, puede decirse que las estrategias de paz noviolentas hacen parte de una metodología permeada de valores como el respeto por el otro, la solidaridad, el perdón y la reconciliación.

\section{Conclusiones}

Hay muchas paces, actores y formas diversas de hacerlas. En algún punto de este artículo se mencionó la Paz Negativa, la Paz Positiva, la Paz Imperfecta, las dos primeras son las que más atención tienen en la academia y con las que se busca resolver los conflictos en el mundo (cfr. Paffenholz, 2013, p.5). No obstante, la Paz Imperfecta abre la posibilidad a la población civil desde distintas contribuciones a la finalización de los conflictos que no son solo bélicos, que han permeado la subjetividad y la vida cotidiana. Esta forma de abordar la paz da cabida a las mujeres que han sufrido la violencia para que sus intervenciones desde metodologías noviolentas sean tenidas en cuenta en la suma de iniciativas que se necesitan para que en Colombia se consolide la paz.

En ese sentido, una comprensión de la paz abierta a la población civil y de condición imperfecta, vincula a las mujeres que sufrieron la violencia y asume como prioridad los procesos de sanación necesarios para su recuperación. Las cuatro iniciativas abordadas en este texto, consideradas como estrategias psicosociales para la sanación, señalan que las mujeres no sólo han contribuido a su propia recuperación, sino que han cohesionado y fortalecido el tejido social del cual hacen parte, en encuentros formales y otros espontáneos donde tiene lugar la complicidad, la confianza, la familiaridad, la solidaridad, el perdón y la reconciliación; valores todos ellos claves para darle solidez a relaciones deterioradas durante tantos años de conflicto armado en el país.

Las estrategias noviolentas asumidas por las mujeres cuestionan a las formas como se ha venido intentando resolver el conflicto, situadas desde lugares dicotómicos, que enraízan profundas brechas de género y ubican desde allí posibilidades para vulnerar los cuerpos y amenazar la propia existencia. Las mujeres y sus luchas recalcan que la paz no se consigue venciendo a los contrincantes, en ese sentido, las mujeres enseñan con sus movimientos políticos y sociales, que la violencia no se promueve, sino que se elimina y que ellas tienen un papel muy importante en dicha tarea. 


\section{Referencias bibliográficas}

Aguilar, M. (2015). El flagelo silencioso de las mujeres en el conflicto armado. DISSÊRTUM.

Andrade Salazar, J. A., Alvis Barranco, L., Jiménez Ruiz, L. K., Redondo Marín, M. P., \& Rodríguez González, L. (2017). La vulnerabilidad de la mujer en la guerra y su papel en el posconflicto. Agora U.S.B., 17(1), 308. https://doi.org/10.21500/16578031.2827

Binazzi, A. (2019). Género y migraciones forzosas en Centroamérica y el Caribe. Una mirada antropológica para los derechos humanos de las niñas, adolescentes y de las mujeres. Collectivus, Revista de Ciencias Sociales, 6(1), 155-176. DOI: http://dx.doi.org/10.15648/Coll.1.2019.9

Bustamante, D. (2015). El proceso de adaptación judicial hacia el povillas conflicto: Decisiones constitucionales con enfoque de género. Opinión Jurídica, (14), 19-36.

Butler, J. (2016). Actos performativos y constitución del género : un ensayo sobre fenomenología y teoría feminista (Johns Hopkins University Press). Retrieved from http://capacitacion.hcdn.gob.ar/wp-content/ uploads/2015/12/actos-Buttler-1.pdf

Cadavid, P. (2014). Mujer: blanco del conflicto armado en Colombia. Analecta Política, 4(7), 301-318. Retrieved from file:///C:/Users/Usuario/Downloads/Dialnet-Mujer-5206403.pdf

Castaño, O. (2013). Conflictos Armados y Construcción de Paz. De la Teorígarrido Vidal a a las Políticas Internacionales de Paz en la Posguerra Fría. Ra Ximhai, 9(2), 69-104. Retrieved from http://uaim.mx/ webraximhai/Ej-27articulosPDF/3-OscarMauricio.pdf

Castrellón Pérez, M., \& Romero Cristancho, C. (2016). Enfoque de género en la implementación de la Ley de Víctimas y Restitución de Tierras: una propuesta para la caracterización de las mujeres y niñas víctimas del conflicto armado en Colombia. (19), 69-113. https://doi.org/10.18046/recs.i19.2166

Céspedes-Báez, L. M., \& Jaramillo Ruiz, F. (2018). 'Peace without women does not go!' Women's struggle for inclusion in Colombia's peace process with the FARC. Colombia Internacional, 94, 83-109. https://doi. org/10.7440/colombiaint94.2018.04

CHCV. (2015). Contribución al entendimiento del conflicto armado en Colombia.

CNMH. (2017). La guerra inscrita en el cuerpo. Centro Nacional de Memoria Histórica.

Fouskas, T., Gikopoulou, P., Ioannidi, E., y Koulierakis, G. (2019). Gender, transnational female migration and domestic work in greece: an intersectional review of research on female migrants' access to labour, healthcare and community associations. Collectivus, Revista de Ciencias Sociales, 6(1), 99-134. http://dx.doi. org/10.15648/Coll.1.2019.7

Garrido, J., \& Vidal, C. (2018). Impulsando el protagonismo de las mujeres en el proceso de paz de Colombia. Polipapers, (2). https://doi.org/10.4995/citecma.2018.9856

Heridas, S., \& Mujeres, E. (2016). Sanando Heridas Entre Mujeres. Experiencias de recuperación psicosocial y construcción de paz de mujeres colombianas. Corporación Sisma Mujer.

Históric, C. N. de M. (2001). Mujeres y guerra Victimas y resistentes en el Caribe Colombiano. Tauro.

Magallón, C. (2004). Las mujeres como sujeto colectivo de construcción de paz. Cuadernos Bakeaz, (61). 
Meza, M. (2015). Capacidades locales para la paz. Apuesta metodológica. Retrieved July 22, 2020, from Fundación Ideas para la Paz website: www.ladoamable.com

Mujeres, A. regional de. (2013). AMOR, 20 años construyendo escenarios de vida. Retrieved from http:// amoroa.blogspot.com/

Muñoz, F. A. (2014). la paz imperfecta. UGR, 392-434. Retrieved from www.ugr.es/ fmunoz/documentos/ pimunozespañol.pdf

ONU, M. (2016). El género y el papel de las mujeres en el proceso de paz.

Paffenholz, T. (2013). International peacebuilding goes local: analysing Lederach's conflict transformation theory and its ambivalent encounter with 20 years of practice. Peacebuilding, 11-27. Retrieved from http:// www.tandfonline.com/loi/rpcb

Paz, A. C. para la. (2016). Acuerdo final para la terminación del conflicto y la construcción de una paz estable y duradera. Retrieved from https://www.cancilleria.gov.co/sites/default/files/ Fotos2016/12.11_1.2016nuevoacuerdofinal.pdf

Rettberg, A. (2013). La construcción de paz bajo la lupa. Estudios Políticos, (42), 13-36. Retrieved from https:// revistas.udea.edu.co/index.php/estudiospoliticos/article/view/15782/13678

Rettberg, A., \& Quishpe, R. (2017). 1900 iniciativas de paz en Colombia. Paso Colombia. Retrieved from https://pasocolombia.org/recursos/1900-iniciativas-de-paz-en-colombia y de la literatura académica internacional

Sánchez Mora, M., \& Rodríguez Lara, Z. (2015). Acciones colectivas de las organizaciones de mujeres por la paz en Colombia. Revista de Paz y Conflictos, 8(2), 149-177. https://doi.org/10.30827/revpaz.v8i2.3190

Silva Arce, P. E. (2017). Los movimientos sociales, el feminismo comunitario y sus aportes a los procesos de construcción de democracia en Colombia. Ciudad Paz-Ando, 10(1), 59. https://doi.org/10.14483/2422278x.11641

Solares, B. (2008). Un acercamiento a la antropología simbólica de Lluís Duch. En Antropología simbólica y corporeidad cotidiana. Cuadernos de Hermenéutica, (2), 1-247. Retrieved from http://bibliotecavirtual. clacso.org.ar/Mexico/crim-unam/20100429110200/SolaresDuch

Tatián, D. (2006). ¿Qué significa actuar políticamente? Nombres Revista de Filosofia, (20).

Urrutia, D. (2017). Mujeres y construcción de paz en Colombia. Retrieved from Irenees.net website: http:// www.irenees.net/bdf_fiche-analyse-1104_es.html

Víctimas, U. de. (2018). Estrategia de reparación integral a mujeres víctimas de violencia sexual. Unidad Para Las Víctimas.

Villa, D, J. (2016). Perdón y reconciliación: una perspectiva psicosocial desde la noviolencia. Polis Revista Latinoamericana, (43), 1-22.

Villa, J, D., Avendaño, M., \& García, E. (2017). Luchas de las mujeres para la construcción de la paz y sus resistencias a la propagación de la violencia: una mirada desde el conflicto armado colombiano. SOMEPSO, (2), 290-308. 
